\title{
Clima social familiar e ideación suicida en adolescentes del centro pre-universitario de la Universidad Nacional Toribio Rodríguez de Mendoza de Amazonas, Chachapoyas, 2017
}

\author{
Family social climate and suicidal ideation in adolescents of the Pre-university \\ center of the National University Toribio Rodríguez de Mendoza de Amazonas, \\ Chachapoyas, 2017
}

\author{
Fany Yaquelin Sifuentes Zabaleta ${ }^{1}$, Sonia Tejada Muñoz
}

\begin{abstract}
RESUMEN
La presente investigación se realizó con el objetivo de determinar la relación que existe entre clima social familiar e ideación suicida en adolescentes del centro Pre universitario de la Universidad Nacional Toribio Rodríguez de Mendoza de Amazonas, Chachapoyas 2017; fue de enfoque cuantitativo; de nivel relacional; de tipo: observacional; prospectivo; transversal y Analítico. El universo muestral estuvo conformado por 147 adolescentes. Para la recolección de datos se utilizó el método de encuesta, técnica el cuestionario y como instrumento se aplicó el cuestionario de la escala de clima social familiar, con una confiabilidad de 0,89 y validez de 0,80 y la escala de ideación suicida, teniendo una confiabilidad es de 0.81 y validez de 0.80 . Los resultados fueron que del 100\% (147) de adolescentes, el 54.4\% (62) tienen un clima social familiar adecuado y el $45.6 \%$ (67) inadecuado. El 49,7\% (73) presentan una ideación suicida baja, el 27.9\% (41) alto y el 22.4\% (33) medio. Así mismo el 42.2\% (62) presentan un clima social adecuado con una ideación suicida baja; mientras que el 23.8\% (35) tienen un clima social inadecuado con una ideación suicida alta, y un 14.3\% (21) medio. La hipótesis se contrastó mediante la prueba estadística no paramétrica del $\mathrm{Ji}$ - cuadrado cuyo valor fue $\mathrm{X} 2$ calculado $=57,9>$; con $\mathrm{gl}=2 ; \mathrm{p}=0.000<0.05$, contrastando de esta manera la hipótesis Alterna. En conclusión: Si existe relación estadística entre clima social familiar e ideación suicida y en los adolescentes del Centro Pre- universitario de la Universidad Nacional Toribio Rodríguez de Mendoza de Amazonas.
\end{abstract}

Palabras clave: Clima social familiar, ideación suicida, adolescentes.

\section{ABSTRACT}

The present research was carried out with the objective of determining the relationship between family social climate and suicidal ideation in adolescents of the Pre-university center of the National University Toribio Rodríguez de Mendoza de Amazonas, Chachapoyas 2017; it was a quantitative approach; of relational level; of type: observational; prospective; cross; Analytical. The sample universe was made up of 147 adolescents. For data collection, the survey method, the questionnaire technique and the questionnaire of the family social climate scale, with a reliability of 0.89 and validity of 0.80 and the scale of suicidal ideation, were applied as an instrument. having a reliability is 0.81 and validity is 0.80 . The results were that $100 \%$ (147) of adolescents, $54.4 \%$ (62) have an adequate family social climate and 45.6\% (67) inadequate. 49.7\% (73) presented low suicidal ideation, $27.9 \%$ (41) High and 22.4\% (33) medium. Likewise, 42.2\% (62) present an adequate social climate with low suicidal ideation; while $23.8 \%$ (35) have an inadequate social climate with high suicidal ideation, and an average $14.3 \%$ (21). The hypothesis was contrasted by means of the nonparametric statistical test of the Chi - square whose value was $\mathrm{X} 2$ calculated $=57$, with $\mathrm{gl}=2 ; \mathrm{p}=0.000<0.005$, thus contrasting the Alterna hypothesis. In conclusion: If there is a statistical relationship between the family social climate and suicidal ideation and the adolescents of the Pre-University Center of the National University Toribio Rodríguez de Mendoza de Amazonas.

Keywords: Family social climate, suicidal ideation, adolescents.

${ }^{1}$ Licenciada de Enfermería. Egresada de la Facultad de Ciencias de la Salud de la Universidad Nacional Toribio Rodríguez de Mendoza de Amazonas. Correo electrónico:sifuentesfany26@gmail.com

${ }^{2}$ Dra en ciencias de Enfermería, asociada al departamento de salud pública. Asesora de tesis, docente asociada a TC-UNTRM-Amazonas Correo electrónico:Cieloceleste120@hotmail.com 


\section{INTRODUCCIÓN}

La ideación suicida es un fenómeno muy habitual durante la adolescencia que se ha convertido en un problema de gran importancia en salud pública a nivel mundial, en particular en la población adolescente y adultos jóvenes donde constituye actualmente la segunda causa de muerte en la mayoría de países; siendo un factor relevante el clima social familiar en el que el adolescente se desarrolla. Por consiguiente, refiere que la familia puede volverse nociva cuando sin saberlo, involuntariamente, no cumple con su rol, cuando las relaciones entre los individuos que la componen son inadecuadas, cuando existen conflictos asociados a la convivencia familiar, siendo así un factor predisponente para la ideación suicida. (Muñoz, 2006, p. 23)

En el año 2000 se suicidaron casi un millón de personas en el mundo, por lo que este problema se convirtió en la décima tercera causa de muerte. Asimismo, Eguiluz, manifiesta que los adolescentes varones cometen actos suicidas en una proporción cuatro veces mayor que las mujeres, en tanto que éstas intentan suicidarse sin lograrlo en una proporción más frecuente que en los varones. (Eguiluz, 2003, p. 4). Según estimaciones realizadas por la Organización Mundial de la Salud (OMS), se calcula que para el 2020 las víctimas podrían ascender a 1,53 millones y de 10 a 20 veces más personas realizarán intentos de suicidios, es decir aproximadamente una muerte cada 20 segundos y un intento cada 1-2 segundos. (Varela, Castillo, Isaza y Castillo, 2008,p. 1)

En nuestro país en el año 2016 se han registrado 241 suicidios, 22 de ellos corresponden a niños y adolescentes de 8 a 17 años. Durante 2012 se presentaron 305 muertes por suicidio, el 18 por ciento correspondieron a niños y adolescentes y sin contar con los intentos suicidas que triplican la cantidad siendo la causa principal las familias disfuncionales, la ausencia de calidez familiar, falta de comunicación con los padres y discordia familiar hacen que existan oportunidades limitadas para el aprendizaje de resolución de problemas. Esta dinámica familiar desfavorable incrementa el riesgo en el intento suicida. (Ministerio de Salud, Perú, 2013, p. 12)

En la región de Amazonas, Chachapoyas en el 2011 se registraron 842 personas que intentaron suicidarse, mientras que en el 2012 hubo 64 casos y el 2014 termino con 7 casos de intento de suicidio sin contar los casos que llegaron a la muerte (Dirección
Regional de Salud de Amazonas, 2016, p. 2). Asimismo, en cada oportunidad que se visitaba al hospital regional Virgen de Fátima en las prácticas hospitalarias se encontraba casos de intento de suicidio y al interactuar con algunos de los adolescentes hospitalizados ellos revelaban "tengo problemas en mi casa con mis padres", "nadie me entiende" "no me siento útil con mi familia"; entre otras expresiones que evidencian un entorno familiar negativo en la vida del adolescente.

Esta situación permitió abordar esta problemática en una población vulnerable; toda vez que en éste grupo etario se agudiza el desequilibrio físico, mental y social acompañado del desajuste en su funcionamiento familiar que genera conflictos determinantes para la ideación suicida en los adolescentes, por lo que se plantea la siguiente pregunta de investigación: ¿Existe relación entre clima social familiar e ideación suicida en adolescentes del Centro Pre-Universitario de la Universidad Nacional Toribio Rodríguez de Mendoza de Amazonas?, teniendo en cuenta que los que se preparan en este Centro Pre-Universitario en su mayoría son adolescentes los cuales en un futuro van a ser líderes del mañana; por lo tanto, se investigó si estos factores pueden afectar en su desarrollo disminuyendo así la calidad de vida e impidiendo tener una salud óptima.

Frente a este problema se planteó como objetivo general: Determinar la relación que existe entre clima social familiar e ideación suicida y en los adolescentes del Centro Pre-Universitario de la Universidad nacional Toribio Rodríguez de Mendoza de Amazonas, Chachapoyas 2017. Por 1o anteriormente expuesto, se considera relevante la realización de la presente investigación, en virtud que sus resultados permiten sustentar intervenciones educativas con un carácter activo, permanente, equitativo y actividades de prevención, con la finalidad reducir el riesgo de ideación suicida que están expuestos los adolescentes del centro Preuniversitario de la Universidad nacional Toribio Rodríguez de Mendoza de Amazonas.

\section{MATERIAL Y MÉTODO}

Es de enfoque cuantitativo; de nivel relacional; de tipo de investigación: observacional; según la planificación de la toma de datos es prospectivo, según el nivel de medición de las variables es de tipo transversal y según el nivel de interés de las variables estadísticas es analíticas. 


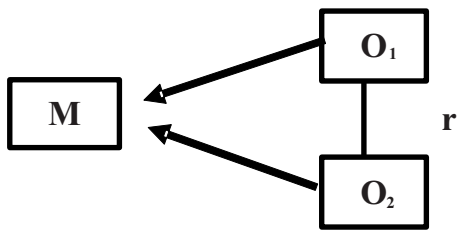

Donde:

$\mathrm{M}=$ La muestra de estudio.

$\mathrm{O}_{1}=$ Medición de la variable $=$ Clima Social familiar.

$\mathrm{O}_{2}=$ Medición de la variable $=$ Ideación Suicida

La Población en estudio fueron 480 adolescentes del centro-preuniversitario matriculados en el Ciclo de Abril a Julio del 2017. La muestra estuvo conformada por 147 adolescentes (considerado entre los 10 a 19 años según la organización mundial de la salud) del centro-preuniversitario. El método fue la encuesta y la técnica del cuestionario y los instrumentos utilizados fueron las escalas de clima social en la familia y la escala de ideación suicida. Se utilizó la estadística descriptiva e inferencial, Ji cuadrado y se procesaron los datos en el SPSS V-21.

\section{RESULTADOS}

Tabla 01: Clima social familiar en los adolescentes del Centro Pre-Universitario de la Universidad Nacional Toribio Rodríguez de Mendoza de Amazonas.

\begin{tabular}{ccc}
\hline $\begin{array}{c}\text { CLIMA SOCIAL } \\
\text { FAMILIAR }\end{array}$ & fi & \% \\
\hline Inadecuado & 67 & 45.6 \\
Adecuado & 80 & 54.4 \\
\hline Total & $\mathbf{1 4 7}$ & $\mathbf{1 0 0 . 0}$ \\
\hline
\end{tabular}

Fuente: Escala del Clima social de la familia aplicado a los adolescentes del Centro Pre Universitario.

Del 100\%(147) de los adolescentes del Centro Pre Universitario el 54.4\% (80) viven en un clima social familiar adecuado y el $45.6 \%(67)$ en un clima familiar inadecuado.

Tabla 02: Ideación suicida en los adolescentes del Centro Pre-Universitario de la Universidad Nacional Toribio Rodríguez de Mendoza de Amazonas

\begin{tabular}{ccc}
\hline IDEACIÓN SUICIDA & $\mathbf{f i}$ & $\mathbf{\%}$ \\
\hline Baja & 73 & 49.7 \\
Media & 33 & 22.4 \\
Alta & 41 & 27.9 \\
\hline Total & $\mathbf{1 4 7}$ & $\mathbf{1 0 0 . 0}$ \\
\hline
\end{tabular}

Fuente: Escala del Clima social de la familia aplicado a los adolescentes del Centro Pre Universitario.
Del 100\% (147) de los adolescentes del Centro Pre Universitario el 49.7\%(73) tiene una ideación suicida baja, el 22.4\%(33) media y el 27.9\%(41) alta.

Tabla 03: Clima social familiar y su relación con ideación suicida en los adolescentes del Centro Preuniversitario de la Universidad Nacional Toribio Rodríguez de Mendoza.

\begin{tabular}{|c|c|c|c|c|c|c|c|c|}
\hline \multirow{3}{*}{$\begin{array}{c}\text { CLIMA } \\
\text { SOCIAL } \\
\text { FAMILIAR }\end{array}$} & \multicolumn{6}{|c|}{ IDEACIÓN SUICIDA } & & \\
\hline & \multicolumn{2}{|c|}{ Bajo } & \multicolumn{2}{|c|}{ Medio } & \multicolumn{2}{|c|}{ Alto } & \multicolumn{2}{|c|}{ TOTAL } \\
\hline & fi & $\%$ & $\mathrm{fi}$ & $\%$ & fi & $\%$ & fi & $\%$ \\
\hline Inadecuado & 11 & 7.5 & 21 & 14.3 & 35 & 23.8 & 67 & 45.6 \\
\hline Adecuado & 62 & 42.2 & 12 & 8.2 & 6 & 4.1 & 80 & 54.4 \\
\hline TOTAL & 73 & 49.7 & 33 & 22.4 & 41 & 27.9 & 147 & 100 \\
\hline
\end{tabular}

Fuente: Clima social en la familia y su relación con ideación suicida.

$\mathrm{X}_{\text {calculado }}=57,9 ;$ con $\mathrm{gl}=2 ; \mathrm{p}=0.000<0.05$

Del 100\% (147) de adolescentes encuestados el $54.4 \%$ (80) viven en un clima social adecuado y presentan ideación suicida nivel bajo en un $42.2 \%$, medio $8.2 \%$, alto $4.1 \%$ y cuando viven en un clima social inadecuado presentan aún más la ideación suicida tanto en el nivel bajo $7.5 \%$, medio $14.3 \%$, alto $23.8 \%$.

\section{DISCUSIÓN}

En la tabla 01, se observa que del 100\% de los adolescentes del Centro Pre Universitario el 54.4\% tiene un clima social familiar adecuado expresando que la familia tiene una buena relación, donde los miembros se apoyan entre sí, expresan directamente sus sentimientos y tienen un buen manejo de conflictos, de la misma manera evalúan la importancia del desarrollo personal en su autonomía para la toma de sus propias decisiones, también se interesan en las actividades intelectuales, culturales, morales y religiosas, no dejando de lado su estabilidad, su organización estructura y control basada en reglas y procedimientos establecidos para una propicia convivencia; de la mima manera existe una $45.6 \%$ que viven en un clima social inadecuado.

Guerrero (2014), dice que no existen diferencia significativa entre las dimensiones de relaciones, desarrollo y estabilidad según género. Varones y mujeres perciben que las interacciones en sus familias en las diversas dimensiones del clima social familiar no presentan variaciones.

Por lo antes discutido se puede explicar que se encontraron similares resultados en esta 
investigación ya que el adolescente independiente del tipo de género al que pertenece perciben de la misma manera un clima social familiar adecuado e inadecuado. Pero lo que los resultados nos explican que los adolescentes de 17 y18 años de edad presentan un mejor clima social familiar.

Guadalupe (2013), demuestran que en la dimensión relaciones el $41.3 \%$ tienen clima social familiar inadecuado y $56.9 \%$ adecuada; para la dimensión desarrollo el 51.8\% tienen clima social familiar inadecuado y el $48.2 \%$ adecuado y en la dimensión estabilidad el $39.4 \%$ tienen clima social familiar inadecuado y el $60.6 \%$ adecuado; existiendo similitud con este trabajo ya que en la dimensión de relaciones tiene un clima social familiar inadecuado.

La familia posibilita el proceso de desarrollo de las habilidades sociales en sus hijos e hijas mediante diversos mecanismos y estrategias, donde juega un papel esencial el ambiente presente en el núcleo familiar (Gonzales,2009, p. 12), pero se puede decir que un clima social familiar tanto adecuado como inadecuado se presenta en todos los tipos de familia como se muestra en este estudio, resaltando un porcentaje mayor en el tipo de familia apartada con un clima social familiar inadecuado al resto de tipos de familias pero proporcionalmente también existe en un clima social adecuado.

En la tabla 02, se observa que del $100 \%$ de los adolescentes del Centro Pre Universitario el $49.7 \%$ tiene una ideación suicida baja, el $27.9 \%$ alta y el $22.4 \%$ media. Un adolescente con una ideación suicida baja puede empezar a teniendo manifestaciones vagas de poco valor en la vida apegándose lentamente a la muerte, con sus temores, sus falsas expectativas de la muerte, de esta manera sus pensamientos suicidas se intensifican cuando se enfrentan a una serie de acontecimientos personales o familiares que en su momento son insoportables, es ahí donde se forja un plan estratégico que tenga como fin acabar con su vida, teniendo un sentimiento de desesperanza, el cual no les permite tener ninguna expectativa a futuro logrando que el adolescente se sienta solo y su tentativa del acto suicida aumente.

Aguirre, et al. (2013), concluyó que el 16.5\% de los estudiantes presentó riesgo suicida; el acoso entre pares manifestó un índice de intensidad entre alta y muy alta de $58.1 \%$; el $38.5 \%$ de la población tuvo buena funcionalidad familiar; el $18 \%$ presentó depresión; la baja autoestima se encontró en 19.6\%; el $11.8 \%$ evidenció dependencia alcohólica; el $23.3 \%$ mostró trastorno de conducta alimentaria, y el consumo de sustancias psicoactivas y cigarrillo fue de $31.4 \%$ y $13.4 \%$, respectivamente.
Leal y Vásquez (2012), afirmó respecto a la ideación suicida que resulta significativo el porcentaje de adolescentes que: manifestaron que alguna vez en su vida experimentaron deseos de estar muerto (38.5\%). De igual modo, cerca de una quinta parte de los adolescentes manifestaron experimentar el deseo de estar muerto alguna vez en el último mes (21.4\%). Porcentajes también altos se hallaron respecto a los adolescentes que pensaron que no vale la pena vivir (prevalencia vida $=31.3 \%$ y prevalencia mes $=$ $18.1 \%$ ). De otro lado, se halló que $28.1 \%$ de los adolescentes entrevistados pensó en suicidarse alguna vez en sus vidas; mientras que $16.5 \%$, lo pensó durante el último mes.

Rojas y Saavedra (2006), presentó la prevalencia de vida respecto al deseo de morir en un $26,7 \%$, pensamiento suicida $10,8 \%$ y planeamiento suicida $4,2 \%$. El deseo de morir fue significativamente mayor en las mujeres $(35,0 \%)$ en comparación con los varones $(15,9 \%)$; lo cual es opuesto a los resultados obtenidos por García. (2006), donde halló que el $68.1 \%$ de los hombres que intentaron suicidarse, el $47.8 \%$ logró consumarlo. En cuanto a las mujeres, el $31.8 \%$ lo intentó y el $9.2 \%$ lo consumaron donde los grupos de edad que presentaron más intentos de suicidio fueron el de 25 a 29 años y el de 15 a 19 años (19\% y 18\%, respectivamente). El grupo en el que se llevaron a cabo más suicidios fue el de 20 y 24 años (10\%).

Por lo antes discutido se puede explicar que se encontraron similares resultados en esta investigación ya que se tuvo un porcentaje mayor de adolescentes entre 17 y 18 años de edad que tiene ideación suicida en sus diferentes niveles, pero lo que llamó la atención fue un porcentaje mayor en el nivel alto; así mismo existe ideación suicida en todos los niveles y en todos los grupos de edad, dato que coincide con lo expresado por la Organización Mundial de la Salud (OMS, 2014, p.02) quien reporta que el suicidio es una de las principales causas de muerte entre la población adolescente.

En la tabla 03, se observa que del $100 \%$ de adolescentes encuestados el $54.4 \%$ viven en un clima social adecuado y presentan ideación suicida baja en un $42.2 \%$, medio $8.2 \%$, alto $4.1 \%$ y cuando viven en un clima social inadecuado presentan aún más la ideación suicida tanto en el nivel bajo $7.5 \%$, medio $14.3 \%$, alto $23.8 \%$.

No teniendo más resultados para constatar la hipótesis, la siguiente tabla de contingencia fue sometida a la prueba estadística no paramétrica Chi cuadrado cuyo valor fue X2calculado $=57,9$; con $\mathrm{gl}=2 ; \mathrm{p}=0.000<0.05$, demostrando de esta manera la hipótesis alterna, lo cual significa que existe relación 
entre ambas variables.

Forero, Siabato, y Salamanca, (2017), indica que el $30 \%$ de los individuos participantes refirieron un nivel alto de ideación suicida, el $67 \%$ un buen funcionamiento familiar, y el $84 \%$ consumo de alcoholde bajo riesgo; así mismo, identificamos una asociación mayor entre ideación suicida y el tipo de funcionalidad familiar grave.

Santiago y Arnold, (2016), realizó un estudio donde comprueba que existe correlación entre ideación suicida y clima social familiar los resultados muestran que el $82.9 \%$ de los casos que presentaron ideación suicida estuvo comprendido en el grupo etario adolescente y juvenil. El 20.90\% de los pacientes tenía algún familiar con antecedentes de intento suicidio que empezó con una idea suicida. Un $68.66 \%$ de los pacientes provenían de hogares de padres separados; el $23.88 \%$ vivía con sus padres casados. Asimismo, el diagnóstico psiquiátrico más frecuente fue la depresión en sus distintas formas, registrándose un total de $56.95 \%$, afectando más a las mujeres.

Pérez (2016), reveló que existe correlación estadísticamente significativa entre la ideación suicida y el funcionamiento familiar, concluyendo que, a menor funcionamiento familiar, mayor ideación suicida y viceversa; Además los adolescentes de la muestra presentan un nivel de ideación suicida medio, y un nivel severo de disfunción familiar.

Rojas y Saavedra (2006), concluyó que la prevalencia de vida del deseo de morir fue de $26,7 \%$, pensamiento suicida $10,8 \%$ y planeamiento suicida $4,2 \%$. Hubo una asociación estadísticamente significativa e inversa entre los indicadores de cohesión familiar y todos los indicadores suicidas; demostrando una relación inversa entre la ideación suicida y la cohesión familiar.

Eguiluz (2003), revelo que las correlaciones entre la escala de ideación suicida y clima social familiar fueron negativas, esto significa que cuando en las áreas de clima familiar se obtienen puntajes altos, los puntajes en Ideación suicida son bajos; sin embargo, cabe resaltar que en esta población las personas que presentan mayor riesgo de ideación suicida fueron los sujetos de sexo masculino.

Por lo antes discutido se puede decir que se encontró similares resultados teniendo un mayor porcentaje de adolescentes de sexo masculino con ideación suicida en comparación con el sexo femenino. Por lo tanto, a través de estos datos se muestra que la variable ideación suicida afecta en mayor medida al sexo masculino en los adolescentes de esta institución educativa. Resaltando que los adolescentes del centro pre universitario que viven en un clima social adecuado presentan una ideación suicida de nivel bajo, por lo tanto se puede expresar que un ambiente familiar apropiado es un determinante definitivo del bienestar, actuando como estructurador del comportamiento y está inmerso en una compleja combinación de variables organizacionales, sociales y físicas; sin embargo los adolescentes con clima social familiar inadecuado tienen mayor porcentaje de ideación suicida por lo que se puede decir que, al disminuir las características negativas de las relaciones familiares así como su grado de conflicto podría tener efectos importantes en la disminución de pensamientos suicidas en los adolescentes vulnerables.

\section{CONCLUSIONES}

Existe relación estadística entre clima social familiar e ideación suicida; una gran proporción, de las familias presentan un clima social adecuado resaltando; de la misma manera existe adolescentes que presentan ideación suicida en todos sus niveles siendo más alarmante que existan altos porcentajes en los niveles de medio y alto. Los adolescentes del sexo masculino de 17 y 18 años de edad presentan ideación suicida en el nivel bajo medio y alto proporcionalmente mayor a los del sexo femenino lo que no sucede con el clima social familiar ya que independientemente del tipo de género al que pertenece perciben de la misma manera un clima social familiar adecuado como inadecuado.

\section{REFERENCIAS BIBLIOGRÁFICAS}

Aguirre, D., Cataño, J., Cañón, S., Marín, D., Rodríguez, j., Rosero, L., y otros. (2013). Riesgo suicida y factores asociados en adolescentes de tres colegios de la ciudad de Manizales. Tesis para obtener el grado de licenciatura. Universidad de Manizales. Manizales, Colombia.

Dirección Regional de Salud de Amazonas. (2016). Informe anual estadístico del HRVF: Enfermedades y atenciones. Recuperado de: http://rpp.postmater/dge.gob.pe.

Eguiluz, L. (2003). Ideación suicida en los jóvenes: Prevención y asistencia. Revista Perspectivas Sistémicas.

Forero, I. Siabato, E. \& Salamanca, Y. (2017). Ideación suicida, funcionalidad familiar y 
consumo de alcohol en adolescentes de Colombia. Revista Latinoamericana de Ciencias Sociales, Niñez y Juventud.

Gonzales et. al (2009). Relación que existe entre el clima social familiar y el rendimiento escolar de los alumnos de la institución educativa "San Santiago" Pamporomás. Tesis para obtener el grado de magister en educación con mención en docencia y gestión educativa. Chimbote, Perú. Universidad cesar vallejo.

García, N. (2006). Ideación e intento suicida en estudiantes adolescentes y su relación con el consumo de drogas. Universidad Nacional Autónoma De México. Tesis para obtener el título de licenciada en psicología. México.

Guadalupe, N. (2013). Clima social familiar y rendimiento académico en estudiantes del cuarto grado del nivel secundario, Institución Educativa San Juan de La Libertad, Chachapoyas - 2013. Tesis para obtener el grado de licenciatura. Universidad Nacional Toribio Rodríguez de Mendoza de Amazonas. Chachapoyas. Perú

Guerrero, Y. (2014). Clima social familiar, inteligencia emocional y rendimiento académico de los alumnos de quinto de secundaria de las instituciones educativas públicas de ventanilla. Tesis para optar el grado de magister en psicología Mención en psicología educativa. Universidad nacional mayor de San Marcos Lima Perú.

Leal, R., Vásquez, L. (2012). Ideación suicida en adolescentes cajamarquinos, prevalencia $y$ factores asociados. Informe de investigación. Universidad privada Antonio Guillermo Urrelo. Cajamarca. Perú.

Ministerio de Salud, Perú. (2013). Minsa registra más de 240 suicidios en lo que va del año. Vital. RPP noticias. Recuperado de: http://rpp.pe/vida-y-estilo/salud/minsaregistra-mas-de-240-suicidios-en-lo-queva-del-ano-noticia-629840

Muñoz, J., Pinto, V, Callata, H., \& Napa, N. (2006). Ideación suicida y cohesión familiar en estudiantes preuniversitarios entre $15 y$ 24 años, Lima 2005. Revista Peruana de Medicina Experimental.

Pérez, P. (2016). Funcionamiento familiar e ideación suicida en alumnos de 5to año de educación Secundaria del distrito de San Juan de Miraflores. Universidad césar vallejo. Lima. Perú.

Rojas, I., Saavedra, J. (2006). Cohesión familiar e ideación suicida en adolescentes de la costa peruana en el año 2006. Instituto Nacional de Salud Mental Honorio Delgado - Hideyo Noguchi. Universidad Peruana Cayetano Heredia. Lima, Perú.

Santiago, P., Arnold, F. (2016). Ideación suicida y clima social familiar en estudiantes de una institución educativa estatal de Chiclayo. Tesis para optar el título de licenciatura en psicología. Chiclayo, Perú. Universidad católica santo Toribio de Mogrovejo.

Varela A, Castillo E, Isaza M, Castillo A. (2008). Intento suicida - vigilancia epidemiológica. Cali: Grupo de Salud. 